\title{
Duration of Ebola virus RNA persistence in semen of survivors: population-level estimates and projections
}

RM Eggo ${ }^{1}$, CH Watson ${ }^{1}$, A Camacho ${ }^{1}$, AJ Kucharski ${ }^{1}$, S Funk ${ }^{1}$, WJ Edmunds ${ }^{1}$

1. Department of Infectious Disease Epidemiology, London School of Hygiene and Tropical Medicine, London, United Kingdom

Correspondence: Rosalind M. Eggo (r.eggo@lshtm.ac.uk)

Citation style for this article:

Eggo R, Watson C, Camacho A, Kucharski A, Funk S, Edmunds W. Duration of Ebola virus RNA persistence in semen of survivors: population-level estimates and projections. Euro Surveill. 2015;20(48):pii=30083. DOI: http://dx.doi.org/10.2807/1560-7917.ES.2015.20.48.30083

Ebola virus can persist in semen after recovery, potentially for months, which may impact the duration of enhanced surveillance required after interruption of transmission. We combined recent data on viral RNA persistence with weekly disease incidence to estimate the current number of semen-positive men in affected West African countries. We find the number is low, and since few reported sexual transmission events have occurred, the future risk is also likely low, although sexual health promotion remains critical.

In this study, a negative binomial distribution is fitted to recent data on persistence of Ebola virus (EBOV) RNA in semen after Ebola virus disease (EVD) symptom onset in EVD survivors. Given the prior reported incidence of EVD in each affected region of Guinea, Liberia, and Sierra Leone, the fitted distribution is used to estimate and model the number of men in these countries with EBOV RNA present in semen, in each week since mid-2014. According to this, the total number of EBOV RNA semen-positive individuals in January 2016 would be low ( $n=73 ; 95 \%$ confidence interval $(\mathrm{Cl}): 15-331)$. Since few reported sexual transmission events have been documented [1], the future risk of such transmission is also likely low.

\section{Modelling Ebola virus RNA persistence in semen}

EBOV can persist in immunoprivileged sites within the body after recovery from infection, specifically in semen $[1,2]$. Post-recovery sexual transmission (PRST) of EBOV via semen has been documented in the ongoing West African outbreak [3]. Understanding potential EBOV shedding after recovery is critical to determining duration of surveillance of survivors, and may impact the length of enhanced surveillance following the interruption of transmission in a country; which currently begins 42 days after the final case and lasts 90 days [4]. Sierra Leone is currently the only country in West Africa in the enhanced surveillance period, which began on 7 November 2015 .
In a separate study, ninety three men had one semen test two to 10 months after onset of confirmed EVD symptoms [1]. Samples were tested by reverse transcription-polymerase chain reaction (RT-PCR) for the presence of EBOV RNA. A positive test by RT-PCR means that viral RNA is present, but does not necessarily mean that infectious virus is present in the sample. The number of positive, negative, or indeterminate samples was reported by month since onset of EVD symptoms and we took the number of days to be the mid-point of each month (Table 1 ).

We estimated the probability that semen was no longer RNA positive by the sampling time, where indeterminate samples were treated as negative. We fitted a negative binomial distribution for the semen clearance time, by maximum likelihood (Figure 1). We extrapolate to 85 weeks after symptom onset.

\section{Estimating numbers of men with Ebola virus RNA in semen in West Africa}

The fitted distribution was used to calculate the number of men with semen positive for EBOV RNA present each week, given the prior incidence of EVD in each affected region of Guinea, Liberia, and Sierra Leone.

For each area (prefecture, county or district) in Guinea, Liberia and Sierra Leone, we use confirmed and probable cases available from the World Health Organization (WHO). The WHO patient database, which is curated and more accurate than the situation reports but subject to delay, was used except for recent weeks where we switch to the WHO situation reports (1 January 2014 to 11 October 2015) because counts were more up to date [5]. Except for the Western Area Districts, Sierra Leone (switch date: 3 May 2015; data not shown), the switch date between WHO data sources was defined within the six weeks prior to the publication date of the last week reported (14 October 2015), as the earliest week when the number of cases in the situation report exceeded that in the WHO patient database. 
Fit of a negative binomial distribution to published data on detection of Ebola virus RNA in semen samples

A.

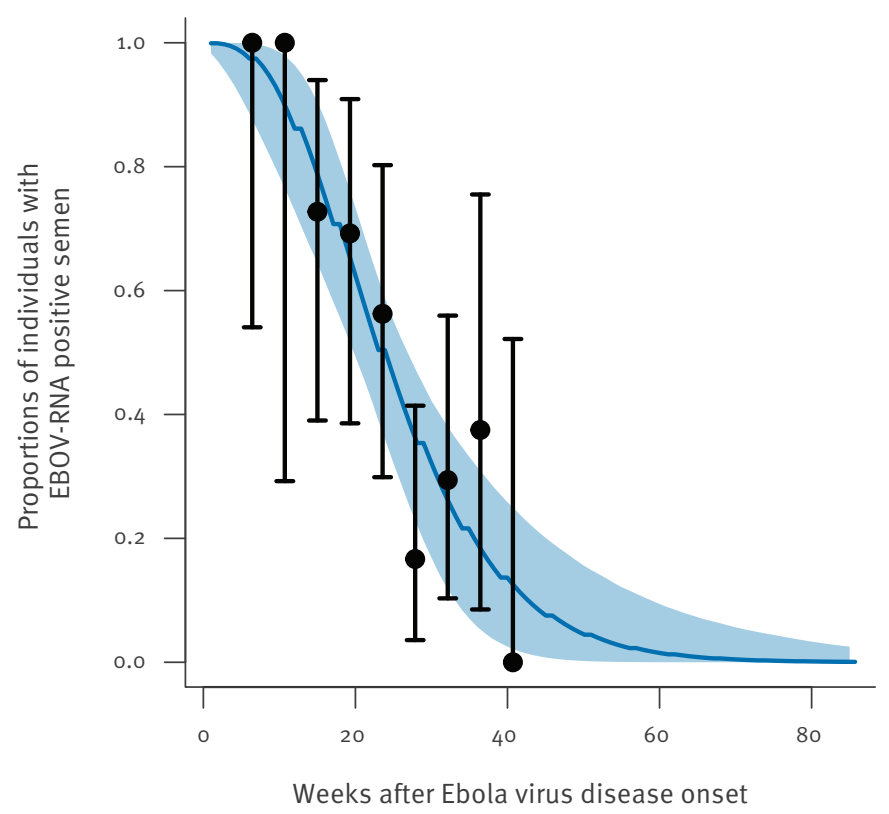

B.

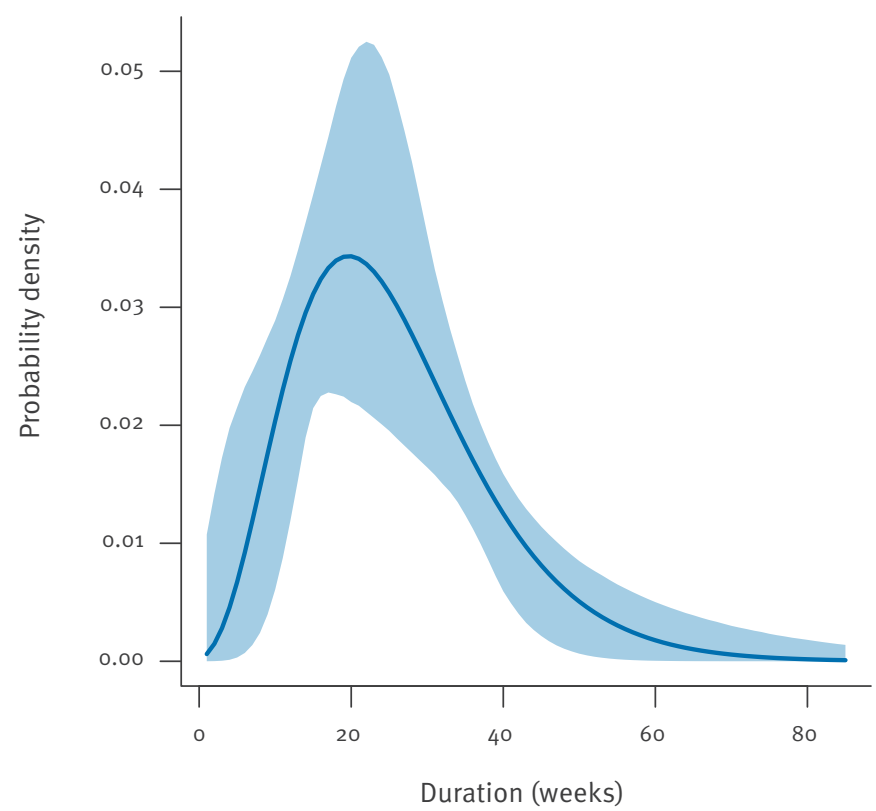

EBOV: Ebola virus.

A. Maximum likelihood (dark blue), 95\% bootstrap interval (shaded) for the fitted distribution from 5,00o bootstrap samples. The negative binomial distribution has parameters size $=4.73$ and probability $=0.16$. Data are shown in black, with a $95 \%$ binomial confidence interval.

B. Maximum likelihood estimate (dark blue) and $95 \%$ bootstrap interval (shaded) of the duration of Ebola virus RNA semen positivity post Ebola virus disease onset.

We assume $60 \%$ case fatality for all weeks, and that $40 \%$ of survivors are male adults [6].

As of 8 November 2015, we estimate that the number of men who may have EBOV RNA-positive semen is 50 (95\% Cl: 22-112), 15 (95\% Cl: 1-84) and 97 (95\% Cl: 24-295) in Guinea, Liberia and Sierra Leone respectively (Figure $2 \mathrm{~A}$ ). The geographical distribution is heterogeneous (Figure $2 \mathrm{~B}$ ) where the region with highest estimated numbers is the Western Area Urban District, Sierra Leone, at 33 (95\% Cl: 8-87). According to our analysis, in the first week of January 2015, the estimated total number of EBOV RNA semen-positive individuals was 2,255 (95\% Cl: 1,946-2,495), in week ending 8 November 2015 was 162 (95\% Cl: 47-491), and in January 2016 will be 73 (95\% Cl: 15-331). Therefore the bulk of person-weeks of risk have passed (Figure $2 \mathrm{~A}$ ), although the infectiousness of EBOV-positive semen over time is not known.

\section{Sensitivity analyses}

Sensitivity of model of RNA persistence in semen to chosen distribution

Due to the low number of samples, and the lack of samples at long time periods, we are unable to determine which parametric distribution fits best (Figure 3). Results above are presented for the negative binomial distribution, and here we examine the sensitivity of our estimates to the chosen distribution. We show the fit to the data (Figure $3 \mathrm{~A}$ ), probability distribution of duration of semen RNA positivity (Figure $3 \mathrm{~B}$ ), and the number of EBOV RNA semen-positive individuals in the first week of January 2015, the week ending 8 November 2015 and the first week of January 2016 for Guinea (Table 2), Liberia (Table 3) and Sierra Leone (Table 4).

In all tested distributions the values for the number of individuals in January 2015 are comparable, as are estimates on 8 November 2015. In estimates for January 2016, the effect of the difference in the tail of the distributions becomes clearer.

\section{Sensitivity to underreporting of estimations of} numbers of men with Ebola virus RNA-positive semen

We tested the sensitivity of our estimates to the assumed fraction of EVD cases that are reported. We show estimates for complete (or 100\% reporting) (as above), constant $70 \%$ reporting, or a rising reporting fraction, beginning at $30 \%$ early in of the epidemic (1 January 2014), reaching 95\% near the end of the 


\section{FIGURE 2}

Estimated numbers of men with semen positive for Ebola virus RNA, in Guinea, Liberia, and Sierra Leone (June 2014-May 2016)

A.

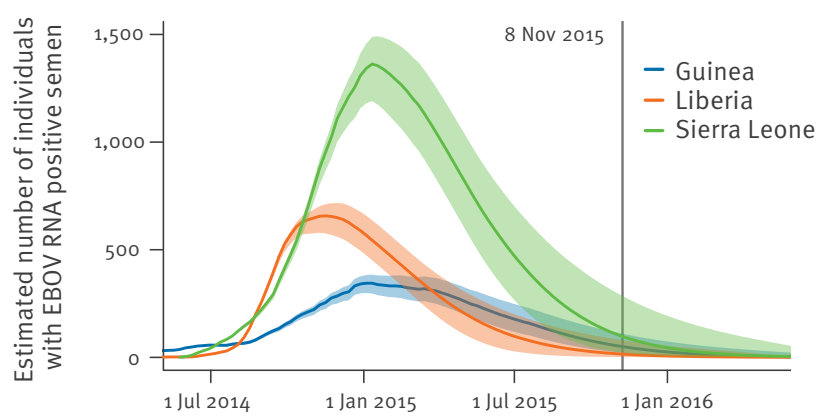

B.

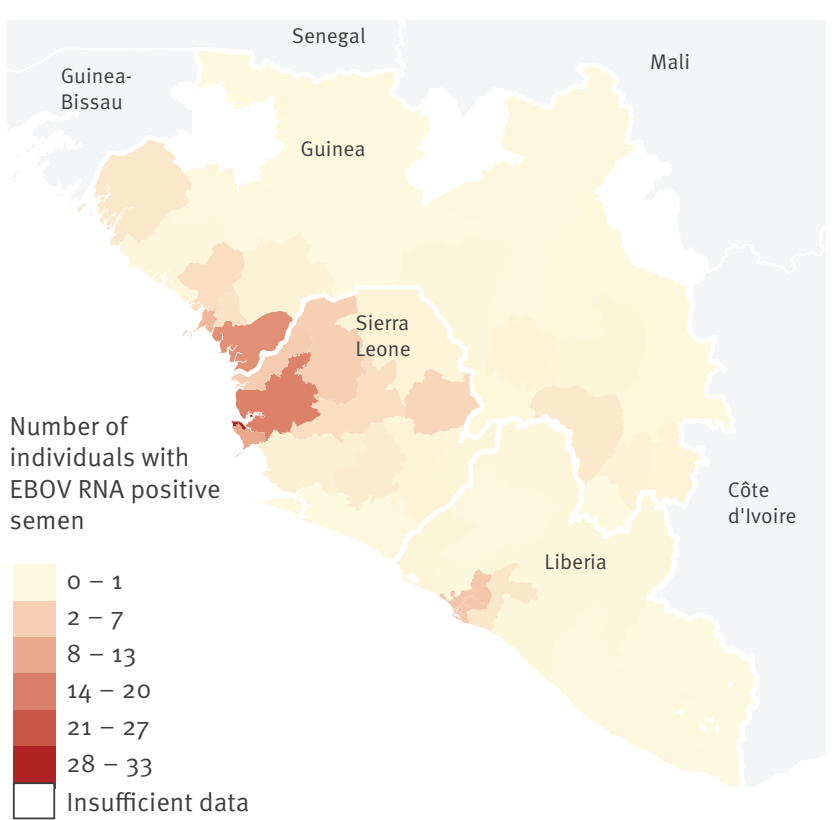

EBOV: Ebola virus.

A. Maximum likelihood (solid line) and $95 \%$ bootstrap interval (shaded) for the number of EBOV RNA semen-positive men in each country. Grey line marks 8 November 2015.

B. Mean number of men with EBOV RNA positive semen in Guinea, Liberia and Sierra Leone by prefecture, county or district as of 8 November 2015.

epidemic (11 October 2015) (Figure 4). Table 5 shows the estimated number in week ending 8 November 2015, which is marked in Figure 4 as a grey line.

Although there are differences between the curves, by November 2015, the vast majority of surviving men would no longer be EBOV RNA semen-positive. Indeed, the number of men remaining is fairly small irrespective of underreporting.

\section{TABLE 1}

Number of initial samples tested and time since Ebola virus disease onset

\begin{tabular}{|l|c|c|c|c|c|}
\hline Month & Days & $\begin{array}{c}\text { Number } \\
\text { Tested }\end{array}$ & Positive & Negative & Indeterminate \\
\hline 1 & 15 & 0 & 0 & 0 & 0 \\
\hline 2 & 45 & 6 & 6 & 0 & 0 \\
\hline 3 & 75 & 3 & 3 & 0 & 0 \\
\hline 4 & 105 & 11 & 8 & 3 & 0 \\
\hline 5 & 135 & 13 & 9 & 1 & 3 \\
\hline 6 & 165 & 16 & 9 & 6 & 1 \\
\hline 7 & 195 & 18 & 3 & 12 & 3 \\
\hline 8 & 225 & 17 & 5 & 10 & 2 \\
\hline 9 & 255 & 8 & 3 & 4 & 1 \\
\hline 10 & 285 & 1 & 0 & 0 & 1 \\
\hline
\end{tabular}

93 samples tested and reported in Deen et al. 2015 [1]. We use the midpoint of the month of testing as number of days.

\section{TABLE 2}

Comparison of predicted number of Ebola virus RNA semen-positive survivors in Guinea on three dates according to the fitted distribution selected

\begin{tabular}{|l|c|c|c|}
\hline \multirow{2}{*}{ Distribution } & 03 Jan 2015 & 08 Nov 2015 & 04 Jan 2016 \\
\cline { 2 - 4 } & Mean $(95 \% \mathrm{CI})$ & Mean (95\% CI) & Mean (95\% CI) \\
\hline Negative binomial & $345(298-382)$ & $50(22-112)$ & $24(8-75)$ \\
\hline Gamma & $349(304-386)$ & $53(24-114)$ & $26(9-77)$ \\
\hline Weibull & $344(298-381)$ & $47(21-115)$ & $22(7-76)$ \\
\hline Logistic & $337(281-379)$ & $45(22-94)$ & $20(8-56)$ \\
\hline Normal & $339(283-381)$ & $41(21-89)$ & $17(7-49)$ \\
\hline Lognormal & $352(309-387)$ & $63(26-131)$ & $36(10-96)$ \\
\hline
\end{tabular}

$\mathrm{Cl}$ : confidence interval.

Mean, lower and upper $95 \% \mathrm{Cls}$ from 5,000 bootstrap samples are given.

\section{TABLE 3}

Comparison of predicted number of Ebola virus RNA semen-positive survivors in Liberia on three dates according to the fitted distribution selected

\begin{tabular}{|l|c|c|c|}
\hline \multirow{2}{*}{ Distribution } & 03 Jan 2015 & 08 Nov 2015 & 04 Jan 2016 \\
\cline { 2 - 4 } & Mean (95\% Cl) & Mean (95\% Cl) & Mean (95\% Cl) \\
\hline Negative binomial & $567(471-657)$ & $15(1-84)$ & $6(0-56)$ \\
\hline Gamma & $577(481-668)$ & $17(2-86)$ & $7(1-58)$ \\
\hline Weibull & $570(475-657)$ & $9(1-85)$ & $3(0-55)$ \\
\hline Logistic & $573(473-663)$ & $9(2-52)$ & $3(1-28)$ \\
\hline Normal & $575(475-664)$ & $4(1-40)$ & $1(0-17)$ \\
\hline Lognormal & $577(481-671)$ & $32(3-116)$ & $19(1-90)$ \\
\hline
\end{tabular}

$\mathrm{Cl}$ : confidence interval.

Mean, lower and upper $95 \% \mathrm{Cls}$ from 5,000 bootstrap samples are given. 
FIGURE 3

Comparison of distributions that can be used to fit the available data on proportions of Ebola virus survivors with semen positive for Ebola virus RNA

A.

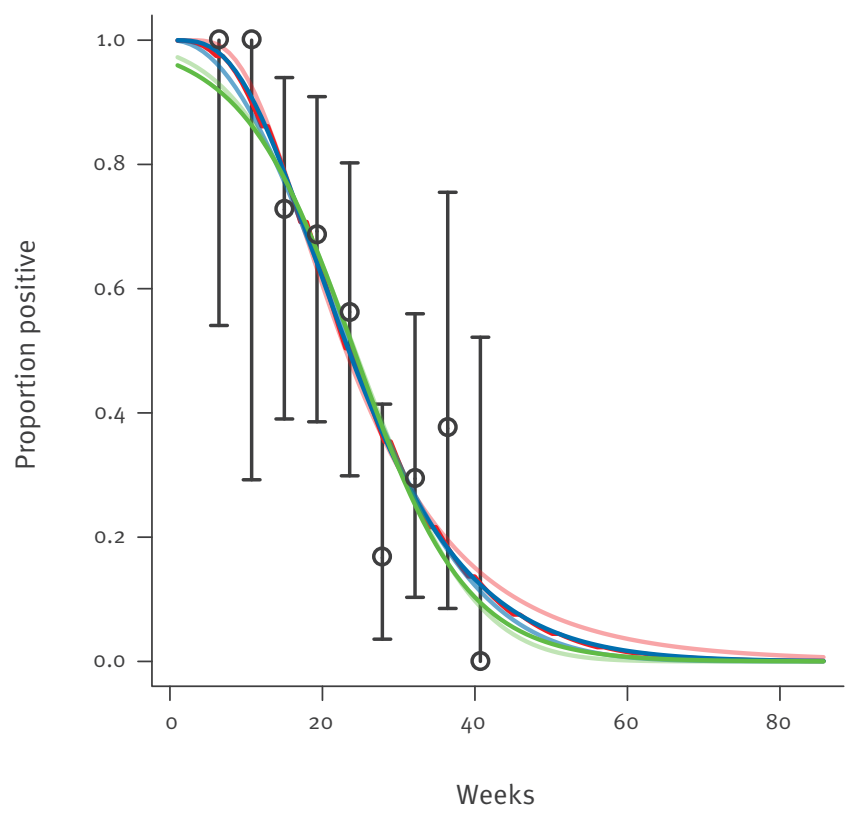

B.

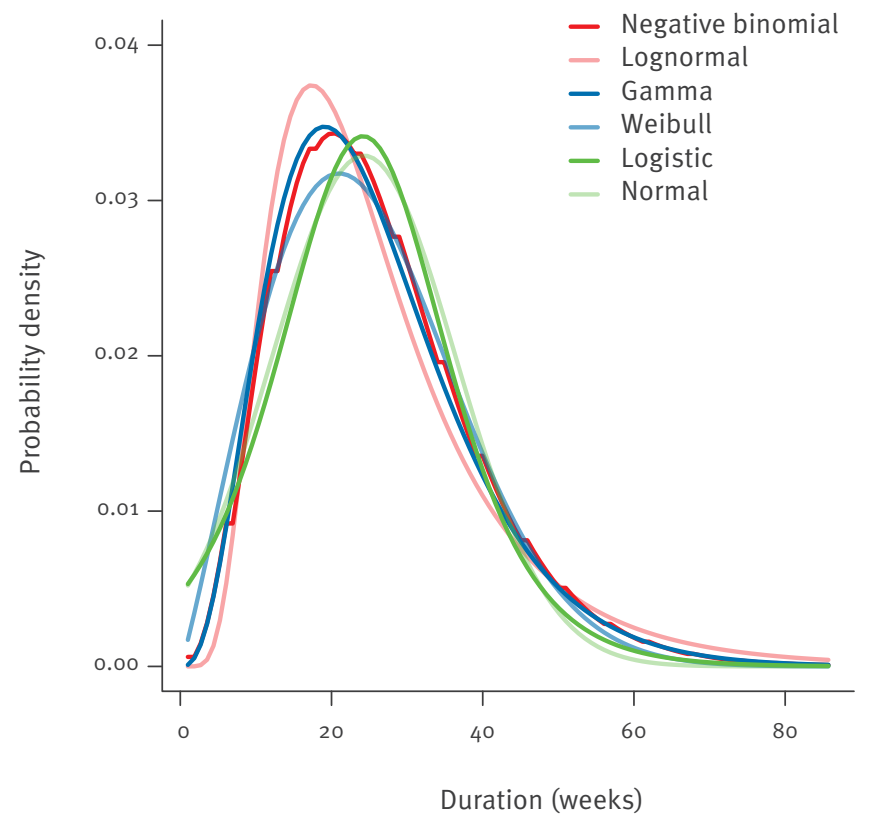

A. Maximum likelihood fit of each distribution is shown with the data (black circles).

B. The probability densities for duration of Ebola virus RNA semen-positivity in weeks are shown for tested distributions.

FIGURE 4

Effect of underreporting of true Ebola virus disease cases on the number of men with semen positive for Ebola virus RNA in Guinea, Liberia and Sierra Leone

A.

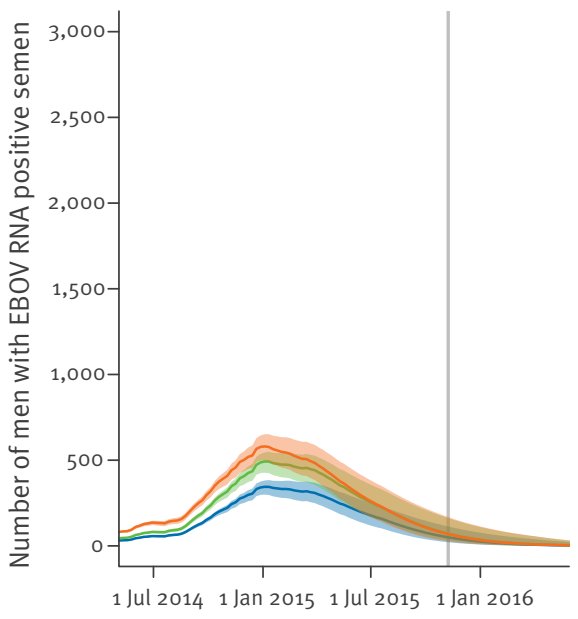

B.

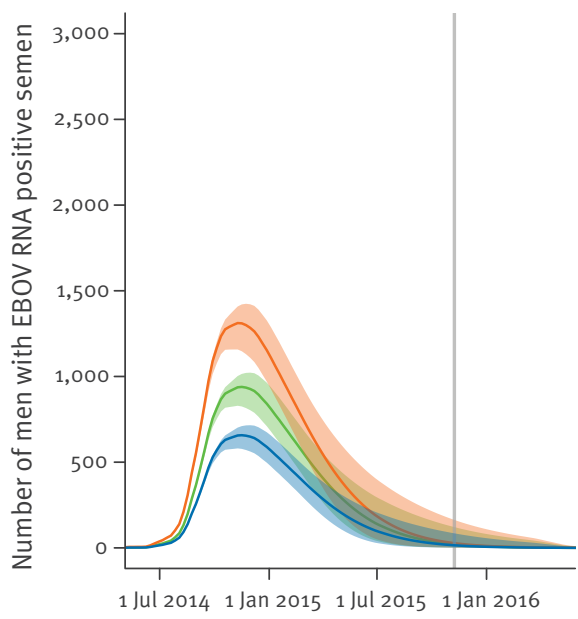

C.

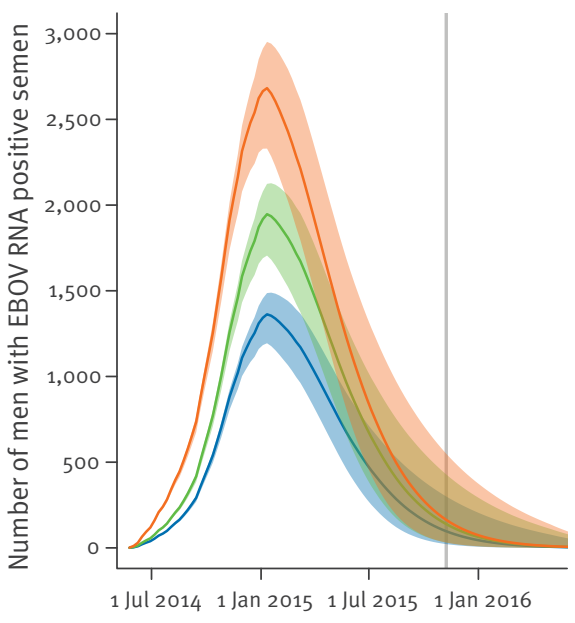

- Complete reporting - $70 \%$ throughout - Starting at $30 \%$, rising to $95 \%$

EBOV: Ebola virus.

Maximum likelihood estimates and $95 \%$ confidence interval $(\mathrm{Cl})$ for the number of EBOV RNA positive men in Guinea (A), Liberia (B), and Sierra Leone (C). Complete (100\%) reporting (blue), constant $70 \%$ reporting rate (green), and a monotonically rising reporting fraction, from $30 \%$ on 1 January 2014 , to $95 \%$ by 11 October 2015 (orange).

4

www.eurosurveillance.org

Check for updates 
TABLE 4

Comparison of predicted numbers of Ebola virus RNA semen-positive survivors in Sierra Leone on three dates according to the fitted distribution selected

\begin{tabular}{|l|c|c|c|}
\hline \multirow{2}{*}{ Distribution } & 03 Jan 2015 & 08 Nov 2015 & 04 Jan 2016 \\
\cline { 2 - 4 } & Mean $(95 \% \mathrm{Cl})$ & Mean $(95 \% \mathrm{Cl})$ & Mean $(95 \% \mathrm{Cl})$ \\
\hline Negative binomial & $1,343(1,177-1,456)$ & $97(24-295)$ & $43(7-200)$ \\
\hline Gamma & $1,360(1,201-1,469)$ & $105(27-302)$ & $49(8-205)$ \\
\hline Weibull & $1,337(1,178-1,449)$ & $83(20-303)$ & $31(6-202)$ \\
\hline Logistic & $1,308(1,109-1,446)$ & $75(23-227)$ & $30(7-129)$ \\
\hline Normal & $1,319(1,114-1,453)$ & $60(19-207)$ & $19(6-104)$ \\
\hline Lognormal & $1,370(1,224-1,473)$ & $143(36-360)$ & $82(13-273)$ \\
\hline
\end{tabular}

$\mathrm{Cl}$ : confidence interval.

Mean, lower and upper $95 \% \mathrm{Cl}$ from 5,000 bootstrap samples are given.

\section{TABLE 5}

Estimated number of men with Ebola virus positive semen in Guinea, Liberia and Sierra Leone, as of 8 November 2015 stratified by the value of underreporting assumed in the data

\begin{tabular}{|l|c|c|}
\hline \multirow{3}{*}{ Country } & Completeness of reporting & Maximum likelihood $(95 \% \mathrm{Cl})$ \\
\hline \multirow{3}{*}{ Guinea } & Complete & $50(21-113)$ \\
\cline { 2 - 3 } & Constant $70 \%$ & $71(30-162)$ \\
\cline { 2 - 3 } & Rising & $68(27-168)$ \\
\hline \multirow{3}{*}{ Sierra Leone } & Complete & $15(1-85)$ \\
\cline { 2 - 3 } & Constant $70 \%$ & $22(2-121)$ \\
\cline { 2 - 3 } & Rising & $28(2-163)$ \\
\cline { 2 - 3 } & Complete & $97(22-300)$ \\
\cline { 2 - 3 } & Constant $70 \%$ & $139(31-428)$ \\
\hline
\end{tabular}

$\mathrm{Cl}$ : confidence interval.

Maximum likelihood estimates, and $95 \%$ bootstrap $\mathrm{Cls}$ are given for complete case identification (100\% reporting), constant $70 \%$ reporting, or a rising reporting fraction, beginning at 30\% early in the epidemic (1 January 2014), reaching $95 \%$ by 11 October 2015.

The maximum duration of EBOV RNA semen positivity is currently unknown, and in our analysis the inferred maximum duration is sensitive to the shape of the decay distribution chosen to fit the available data. The mean number of EBOV RNA semen-positive men at each time point is less sensitive to the choice of distribution, although the confidence intervals vary widely on this assumption.

\section{Discussion}

Our analysis indicates that the number of men remaining in West Africa with EBOV RNA detectable in their semen is currently low and continuing to fall. The geographical distribution of these individuals is heterogeneous, and is related to past incidence levels and the timing of cases in those districts.
To better understand the risk of onward transmission, the relationship between testing positive for EBOV RNA by RT-PCR, and the likelihood of sexual transmission needs quantification. Recent sexual transmission events [3] further highlight the urgent need for comprehensive survivor screening programmes, which include testing of bodily fluids such as semen to determine a more certain time at which RNA is no longer present. Such programmes would not only provide insight into how to protect the intimate contacts of survivors, but can provide reassurance to the many survivors who are likely no longer EBOV semen-positive.

Our base-case estimates do not include suspected cases, underreporting of symptomatic cases or the possibility of EBOV RNA-positive semen in asymptomatic cases. Under these circumstances, the number of EBOV RNA semen-positive individuals is slightly increased (Figure 4). In addition, although we present point estimates for numbers of remaining EBOV RNA semen-positive survivors, the values rely not only on accurate EVD incidence data, but also on assumptions of constant survival rates, as well as statistical uncertainty in the fitting procedure. Therefore, point estimates should be interpreted with care, and attention given to the wide confidence intervals.

Up to 20 suspected PRST events have been reported in West Africa to date [1], although ring vaccination of at-risk contacts in Guinea may have reduced late transmission [7]. Even assuming 20 PRST events to be a substantial underestimate, future numbers may be low. Given this low potential for transmission, we should be cautious not to further stigmatise an already marginalised group. However, since a single PRST event could restart wider transmission, and the duration for which men may remain EBOV semen-positive is long, continued promotion of sexual health and EVD surveillance remain vital.

\section{Acknowledgements}

The authors acknowledge funding from the Innovative Medicines Initiative 2 (IMI2) Joint Undertaking, under grant agreement 115854 (RME, WJE), the Research for Health in Humanitarian Crises ( $22 \mathrm{HC}$ ) Programme, managed by Research for Humanitarian Assistance (Grant 13165) (AC, SF, AJK), and the Norwegian Institute of Public Health (CHW, WJE). IMI2 receives support from the European Union's Horizon 2020 research and innovation programme and the European Federation of Pharmaceutical Industries and Associations (EFPIA).

\section{Conflict of interest}

None declared.

\section{Authors' contribution}

RME and WJE developed the analysis plan. RME implemented the analysis, with contribution from $A C$ and CHW. RME, CHW, WJE, AJK and SF interpreted the results and wrote the paper. 


\section{References}

1. Deen GF, Knust B, Broutet N, Sesay FR, Formenty P, Ross C, et al. Ebola RNA Persistence in Semen of Ebola Virus Disease Survivors - Preliminary Report. N Engl J Med. 2015. DOI: 10.1056/NEJMoa1511410 PMID: 26465681

2. Rowe AK, Bertolli J, Khan AS, Mukunu R, Muyembe-Tamfum JJ, Bressler D, et al. Clinical, virologic, and immunologic followup of convalescent Ebola hemorrhagic fever patients and their household contacts, Kikwit, Democratic Republic of the Congo. Commission de Lutte contre les Epidémies à Kikwit. J Infect Dis. 1999;179(S1) Suppl 1;S28-35. DOI: 10.1086/514318 PMID: 9988162

3. Mate SE, Kugelman JR, Nyenswah TG, Ladner JT, Wiley MR, Cordier-Lassalle T, et al. Molecular Evidence of Sexual Transmission of Ebola Virus. N Engl J Med. 2015. DOI: 10.1056/ NEJMoa1509773 PMID: 26465384

4. World Health Organization (WHO). Criteria for declaring the end of the Ebola outbreak in Guinea, Liberia or Sierra Leone. Geneva: WHO. Available from: http://www.who.int/csr/ disease/ebola/declaration-ebola-end/en/

5. World Health Organization (WHO). WHO Situation Reports Download Page. Geneva: WHO. Available from: http://apps. who.int/gho/data/node.ebola-sitrep.quick-downloads

6. WHO Ebola Response Team,. Ebola virus disease in West Africa--the first 9 months of the epidemic and forward projections.N Engl J Med. 2014;371(16):1481-95. DOI: 10.1056/ NEJMoa1411100 PMID: 25244186

7. Henao-Restrepo AM, Longini IM, Egger M, Dean NE, Edmunds WJ, Camacho A, et al. Efficacy and effectiveness of an rVSVvectored vaccine expressing Ebola surface glycoprotein: interim results from the Guinea ring vaccination clusterrandomised trial. Lancet. 2015;386(9996):857-66. DOI: 10.1016/S0140-6736(15)61117-5 PMID: 26248676 